\title{
Effect of dipole orientation on Purcell factor for the quantum emitter near silicon nanoparticle
}

Anastasia S. Zalogina, Javid Javadzade, Dmitry A. Zuev, Roman S. Savelev, Vadim V. Vorobyov, Sergey V. Makarov, Pavel A. Belov, Alexey V. Akimov, and llya V. Shadrivov

Citation: AIP Conference Proceedings 1874, 040058 (2017); doi: 10.1063/1.4998131

View online: http://dx.doi.org/10.1063/1.4998131

View Table of Contents: http://aip.scitation.org/toc/apc/1874/1

Published by the American Institute of Physics

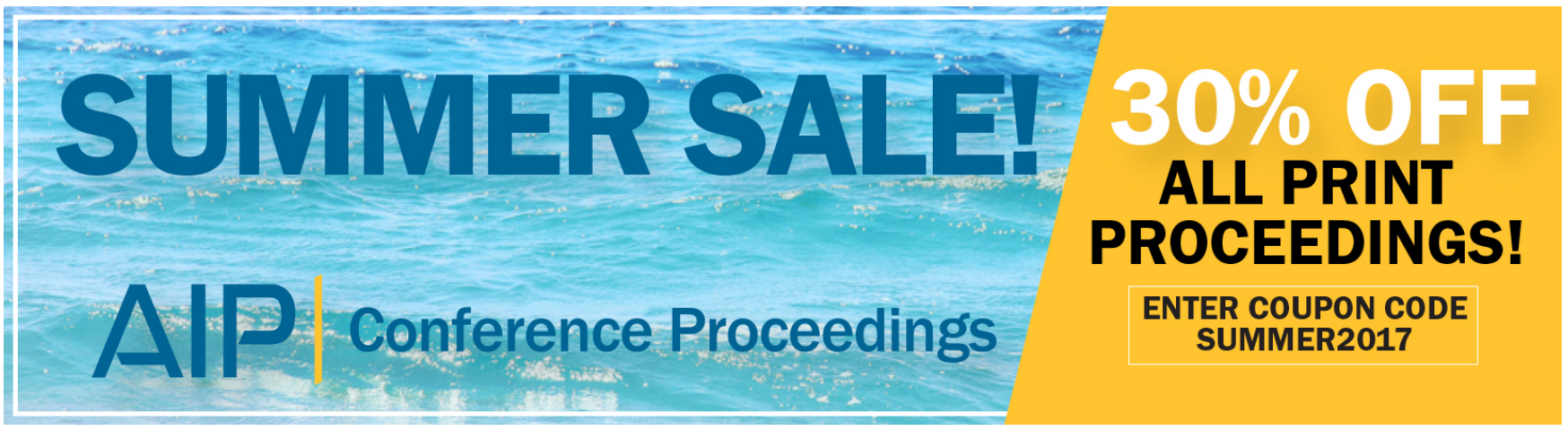




\title{
Effect of Dipole Orientation on Purcell Factor for the Quantum Emitter near Silicon Nanoparticle
}

\author{
Anastasia S. Zalogina ${ }^{1, a)}$, Javid Javadzade ${ }^{2,3}$, Dmitry A. Zuev ${ }^{1, b)}$, Roman S. \\ Savelev ${ }^{1}$, Vadim V. Vorobyov ${ }^{2,4}$, Sergey V. Makarov ${ }^{1}$, Pavel A. Belov ${ }^{1}$, Alexey V. \\ Akimov $^{3,4,5}$ and Ilya V. Shadrivov ${ }^{1,6}$ \\ ${ }^{1}$ Department of Nanophotonics and Metamaterials, ITMO University, 49, Kronverksky pr., 197101, Saint-Petersburg, \\ Russia \\ ${ }^{2}$ Moscow Institute of Physics and Technology, 9, Institutskiy per., 141700, Dolgoprudny, Moscow Region, Russia \\ ${ }^{3}$ Russian Quantum Center, 100A, Novaya str., 143025, Skolkovo, Moscow, Russia \\ ${ }^{4}$ Department of Physics and Astronomy, PN Lebedev Institute RAS, 53, Leninskii pr., 119991, Moscow, Russia \\ ${ }^{5}$ Optics Department, Texas A\&M University, 4242 TAMU, College Station, TX, 77843, USA \\ ${ }^{6}$ Nonlinear Physics Centre, Research School of Physics and Engineering, Australian National University, Canberra \\ ACT 2601, Australia \\ ${ }^{\text {a)} C o r r e s p o n d i n g ~ a u t h o r: ~ a . z a l o g i n a @ m e t a l a b . i f m o . r u ~}$ \\ b)zuewda@gmail.com
}

\begin{abstract}
Understanding the optical properties of quantum emitters is the cornerstone of many phenomena in nanophotonics. Spontaneous emission from quantum emitters can be modified when they are placed in resonators, for example, near plasmonic or dielectric nanoantennas. We numerically study the Purcell factor of the emitter inside a nanodiamond placed near the resonant silicon nanoparticle. We show that in this system the 11-fold enhancement can be achieved for a specific orientation of the dipole moment of the emitter. The obtained results are of particular importance for nanophotonic applications which aim to control optical properties of quantum light sources.
\end{abstract}

\section{INTRODUCTION}

Studies of quantum emitters are of great interest for rapidly developing field of nanophotonic device fabrication. Emission properties of the emitter depend on the optical transition between its atomic levels as well as on the local density of optical states [1,2]. Emission can be accelerated by using the so-called Purcell effect. There are several techniques for achieving the modification of the emitter emission and for the enhancement of Purcell factor: using resonant cavities $[3,4,5]$, waveguides [6,7], photonic crystals $[8,9]$ and nanoantennas $[10,11]$. The latter examples include plasmonic and dielectric nanoparticles. High-index dielectric nanoparticles are are of particular interest due to smaller size, low dissipative losses and presence of magnetic response [12,13].

We will consider quantum systems containing nanodiamonds with nitrogen vacancy (NV) center. Each such center we model as a dipole, and we study its emission rate when it is placed near a dielectric nanoantenna. NV center is the combination of an embedded nitrogen atom and a vacancy in the nanodiamond lattice (see Fig. 1(a)). Such defect is characterized by useful fluorescence properties and its high quantum efficiency as compared to other color centers [14]. The main advantage of application of nanodiamonds with NV center is the presence of zero phonon line (ZPL) in the photoluminescence spectrum. ZPL at the wavelength of $637 \mathrm{~nm}$ in the photoluminescence spectra of nanodiamonds with NV center is explained by optical transition from ground $\left({ }^{3} \mathrm{~A}_{2}\right)$ to excited $\left({ }^{3} \mathrm{E}\right)$ state. At room temperatures, the intrinsic emission limits the collection of photons because of a broad phonon part in the photoluminescence spectrum of nanodiamonds. Moreover orientation of such emitter strongly influences the emission ratio and directivity. To study this problem, we perform numerical simulations that show how the orientation of the dipole in a nanodiamond influences the Purcell factor induced by a silicon nanoparticle placed near ninodiamond.

Proceedings of International Conference on Metamaterials and Nanophotonics (METANANO-2017)

AIP Conf. Proc. 1874, 040058-1-040058-3; doi: 10.1063/1.4998131

Published by AIP Publishing. 978-0-7354-1554-6/\$30.00 


\section{RESULTS AND DISCUSSIONS}

We assume that the NV center is placed in a nanodiamond with the shape of a sphere of diameter $d_{n d}=50 \mathrm{~nm}$ and the refractive index of $\left(n_{n d}=2.4\right)$. Next, we take a silicon nanoparticle of diameter $\left(d_{S i}=140\right) \mathrm{nm}$ and refractive index of $\left(n_{S i}=3.78\right)$. To reflect more realistic geometry, we assume that both particles are placed on a glass substrate $\left(\mathrm{SiO}_{2}\right)$ $\left(n_{\text {sub }}=1.5\right)$.

Numerical calculations of spontaneous emission are performed using commercial software CST Microwave Studio. NV-center is modelled as an electrically small electric dipole. To better understand how silicon nanoparticle modifies emission spectra of NV center in nanodiamonds the study is carried out for two geometries (see Fig. 1(b) and (c)): when nanodiamond is next to the silicon particle, and when it is on top of this particle. Various mutual positions of nanodiamond and silicon nanoparticle on the substrate, as well as the direction of the NV-centre dipole moment influences the electromagnetic interaction between the particles and resulting scattering fields and spontaneous emission rates.

(a)

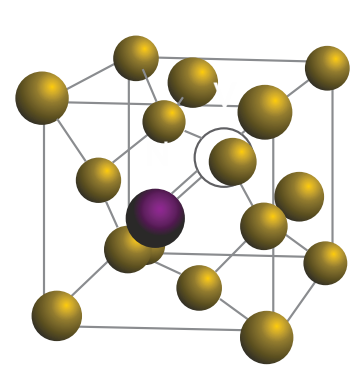

(b)

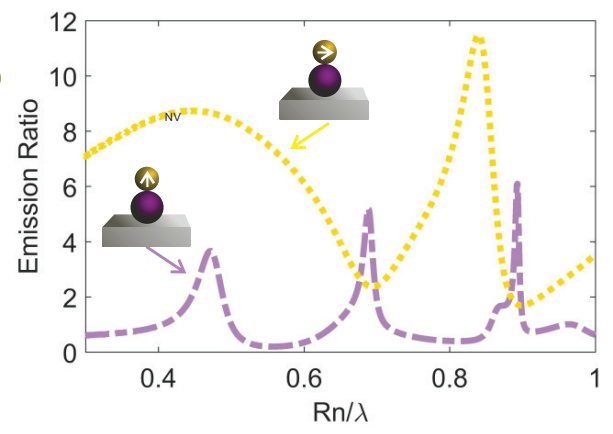

(c)

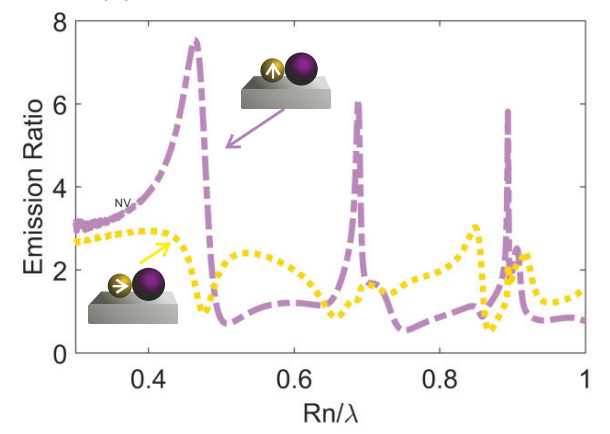

FIGURE 1. (a) Schematic crystallographic structure of the NV center in a diamond. There are the substitution nitrogen atom (purple) and a vacancy (white); Numerically calculated emission rate for two geometries: (b) nanodiamond is on top of the silicon nanoparticle (c) nanodiamond is next to the silicon nanoparticle. Insets in (b) and (c) show schematically the geometries of the studied structures with different orientations of NV center dipole moment.

To study the influence of the high-index nanoparticle on the emitter in the nanodiamond we calculate Purcell factor with and without the silicon nanoparticle using the method of input impedances [15].

For the first studied geometry, the enhancement of emission ratio is predicted to reach values up to 11.5 (see Fig. 1(b)) and for the second geometry the value is up to 7.5 (see Fig. 1(c)). In spite of higher enhancement of the first geometry, the second type of placement of the nanodiamonds near silicon particles is easier to realize experimentally by using, e.g., methods of laser abliation or colloidal chemistry. The enhancement of emission rate by the silicon nanoparticle is caused by the coupling of the radiation of the emitter to the dielectric nanoparticle mode.

The results shown in Fig. 1(b) and (c) demonstrate strong dependence of the Purcell enhancement on the orientation of the dipole. We observe intuitively understandable result that the stronger enhancement in both geometries is obtained when the dipole is oriented perpendicular to the line connecting the centres of the nanodiamond and silicon particle. In this case radiation of the dipole couples stronger to the resonant mode of the silicon nanoparticle justifying larger effect. 


\section{CONCLUSION}

We demonstrate numerically that the Purcell effect depends on the orientation of the dipole emitter in a nanodiamond as well as on the mutual position of the nanodiamond and the silicon resonator. We show that the combination of quantum emitter in the nanodiamond with resonant dielectric nanoparticles provides the enhancement of the emission ratio by up to 11 times. The maximum emission enhancement is achieved when the dipole is oriented perpendicular to the line connecting emitter and the center of the nanoparticle, and at the frequencies close to the nanoparticle resonances. The calculated results will be useful for experimental demonstration of controlling the quantum emitter radiation rate.

\section{ACKNOWLEDGMENTS}

This work was supported by the Russian Science Foundation (Grant No. 16-19-10367).

\section{REFERENCES}

[1] Agio, M. and Cano D.M., "Nano-optics: The Purcell factor of nanoresonators," Nature photonics, Vol. 7, No. 9, 674-675, 2013.

[2] Wang, Q., Stobbe S. and Lodahl P., "Mapping the local density of optical states of a photonic crystal with single quantum dots," Physical review letters, Vol. 107, No. 16, 167404, 2011.

[3] Vahala, K.J. “Optical microcavities,” Nature, Vol. 424, No. 6950, 839-846, 2003.

[4] Reithmaier, J. "Strong coupling in a single quantum dot-semiconductor microcavity system," Nature, Vol. 432, No. 7014, 197-200, 2004.

[5] Gérard, J.M. "Enhanced spontaneous emission by quantum boxes in a monolithic optical microcavity," Physical review letters, Vol. 81, No. 5, 1110, 1998.

[6] Babinec, T.M., Hausmann B.J.M., Khan M., Zhang Y., Maze J.R., Hemmer P.R. and Lončar M., “A diamond nanowire single-photon source," Nature nanotechnology, Vol. 5, No. 3, 195-199, 2010.

[7] Henderson, M.R., Gibson B.C., Ebendorff-Heidepriem H., Kuan K., Afshar V., Orwa J.O., Aharonovich I., Tomljenovic-Hanic S., Greentree A.D., Prawer S., et.al., "Diamond in tellurite glass: a new medium for quantum information," Advanced Materials, Vol. 23, No. 25, 2806-2810, 2011.

[8] Noda, S., Fujita M. and Asano T., "Spontaneous-emission control by photonic crystals and nanocavities," Nature photonics, Vol. 1, No. 8, 449-458, 2007.

[9] Englund, D., Fattal D., Waks E., Solomon G., Zhang B., Nakaoka T., Arakawa Y., Yamamoto Y. and Vučković J., "Controlling the spontaneous emission rate of single quantum dots in a two-dimensional photonic crystal," Physical review letters, Vol. 95, No. 1, 013904, 2005.

[10] Baranov, D.G., Savelev R.S., Li S.V., Krasnok A.E. and Alù A., "Modifying magnetic dipole spontaneous emission with nanophotonic structures," Laser $\mathcal{F}$ Photonics Reviews, 2017.

[11] Schietinger, S., Aichele T., Wang H.Q., Nann T. and Benson O., "Plasmon-enhanced upconversion in single NaYF4: Yb3+/Er3+ codoped nanocrystals,” Nano letters, Vol. 10, No. 1, 134-138, 2009.

[12] Evlyukhin, A. B., Reinhardt C. and Chichkov B.N., "Multipole light scattering by nonspherical nanoparticles in the discrete dipole approximation," Physical Review B, Vol. 84, No. 23, 235429, 2011.

[13] Kuznetsov, A. I., Miroshnichenko A.E., Brongersma M.L., Kivshar Yu.S. and Luk'Yanchuk B., “Optically resonant dielectric nanostructures," Science, Vol. 354, No. 6314, aag2472, 2016.

[14] Aharonovich, I., Englund D. and Toth M., "Solid-state single-photon emitters," Nature Photonics, Vol. 10, No. 10, 631-641, 2016.

[15] Krasnok, A. E., Maloshtan A., Chigrin D.N., Kivshar Yu.S. and Belov P.A., Enhanced emission extraction and selective excitation of NV centers with alldielectric nanoantennas, Laser and Photonics Reviews, Vol. 9, No. 4, 385391, 2015. 